\title{
KORTE AANKONDIGINGEN
}

S. Ullmann, Précis de sémantique française, ze édition (Bibliotheca Romanica) Editions A. Francke, Bern, I962, rel. Fr. S. I9.50.

Il s'agit d'une reproduction photocopiée de la première édition, avec toutefois quelques corrections. Ainsi, une faute d'impression qui rendait incompréhensible le second alinéa de la page 59 ( $p$ pour indiquer $n$ mouillé) a disparu. La bibliographie a été augmentée par l'introduction d'ouvrages parus depuis I952. L'auteur a ajouté I6 pages de notes „pour rectifier ou développer quelques passages et surtout pour mettre le lecteur au courant des importants progrès qui ont été réalisés au cours de ces dernières années". Le livre ayant prouvé son utilité, une troisième édition ne se fera sans doute pas trop attendre. Il serait bon alors d'incorporer les additions au texte et de faire disparaitre les traces par-ci par-là d'une rédaction quelque peu hâtive.

Werner Bahner, Kurze Bibliographie für das Studium der romanischen Sprachwissenschaft mit besonderer Berücksichtigung des Französischen, VEB Max Niemeyer Verlag (Halle Saale), 1962, 6 DM.

Cette bibliographie, qui s'étend sur ro6 pages, est sinon complète, du moins fort abondante. L'éditeur commercial est Niemeyer en Allemagne de l'Est, ce qui explique un certain nombre de titres en langue et en caractères russes. La brochure a ceci de particulier qu'à l'intérieur de chaque section les titres ne se suivent ni en ordre chronologique, ni en ordre alphabétique, mais plutôt en vrac. Ainsi, sous 9 Lehnwortschatz (p. 36) on lit d'abord Wartburg, Der Einflusz ... de 1930, et ensuite seulement $H$. Berger, Die Lehnwörter... de 1899. De même, sous I 7 Syntax (allgemein pp. 43-44) on rencontre d'abord la Syntaxe de C. de Boer parue en 1954, et ensuite son Introduction de I933. On se demande pourquoi.

Ulrich Leo, Interpretaciones hispanoamericanas; ensayos de teoria y práctica estilisticas, 1939-1958. Santiago de Cuba, 1960, XVI, 237 pp.

Ayant commencé ses études romanes en explorant le domaine français, Ulrich Leo se tourna ensuite vers les littératures italienne - on se rappelle son Torquato Tasso, I957et espagnole. Le présent recueil groupe deux séries d'études, essais théoriques et comptes-rendus critiques, parues dans différentes revues de l'Amérique du Sud et portant notamment sur la jeune littérature hispano-américaine.

M. I. GERHARD T.

\section{INHOUD VAN TIJDSCHRIFTEN}

Revue des Langues Vivantes. Tijdschrift voor Levende Talen, Jg.XXVII, No 4, 1962. G. Compère, Maurice Maeterlinck et l'évolution de son théâtre. - Fr. Closset, Gerrit Achterberg. - Michel Vanhelleputte, Wer war Hugo von Hofmannsthal? - J. M. Warland, Morphologie und Syntax der Sprachnamen im Deutschen. - Jean Tromme, A l'intention des stagiaires et des débutants.

id., Jg. XXVIII, No. 5, 1962. G. Hougardy-Debaye, De kwestie van de uitspraak van het Nederlands in de „Handelingen van de Nederlandsche taal- en letterkundige congressen". - R. Leroy, De structuur 'Millioenenstudiën'. - Michel Vanhelleputte A propos de l'influence d'Elkerlijc en Allemagne. - Eugene E. Reed, Franz Kafka: The Futile Messenger. $-\mathrm{R}$. Trousson, A propos de la traduction d'un vers de 'Cimetière Marin'. - J. Dierickx, King and Archbishop. - Robert M. Slabey, Faulkner's 'Mosquitoes' and Joyce's 'Ulysses'. - A. F. Baarslag, La modalité de la parole. Jean Tromme, A l'intention des stagiaires et des débutants. Quelques réflexions sur 'La règle d'or' (I). 